\title{
Bound on 3 + 1 Active-Sterile Neutrino Mixing from the First Four-Week Science Run of KATRIN
}

M. Aker, ${ }^{1}$ K. Altenmüller, ${ }^{2,3}$ A. Beglarian, ${ }^{4}$ J. Behrens, ${ }^{5,6}$ A. Berlev, ${ }^{7}$ U. Besserer, ${ }^{1}$ B. Bieringer, ${ }^{8}$ K. Blaum, ${ }^{9}$ F. Block, ${ }^{5}$ B. Bornschein, ${ }^{1}$ L. Bornschein, ${ }^{6}$ M. Böttcher, ${ }^{8}$ T. Brunst,${ }^{2,10}$ T. S. Caldwell, ${ }^{11,12}$ L. La Cascio, ${ }^{5}$ S. Chilingaryan, ${ }^{4}$ W. Choi, ${ }^{5}$ D. Díaz Barrero, ${ }^{13}$ K. Debowski, ${ }^{14}$ M. Deffert, ${ }^{5}$ M. Descher, ${ }^{5}$ P. J. Doe, ${ }^{15}$ O. Dragoun, ${ }^{16}$ G. Drexlin,,${ }^{5}$ S. Dyba, ${ }^{8}$ F. Edzards ${ }^{2,10}$ K. Eitel, ${ }^{6}$ E. Ellinger,${ }^{14}$ R. Engel, ${ }^{6}$ S. Enomoto, ${ }^{15}$ M. Fedkevych,${ }^{8}$ A. Felden, ${ }^{6}$ J. A. Formaggio, ${ }^{17}$ F. M. Fränkle, ${ }^{6}$ G. B. Franklin, ${ }^{18}$ F. Friedel,${ }^{5}$ A. Fulst,${ }^{8}$ K. Gauda, ${ }^{8}$ W. Gil,${ }^{6}$ F. Glück, ${ }^{6}$ R. Grössle, ${ }^{1}$ R. Gumbsheimer, ${ }^{6}$ T. Höhn, ${ }^{6}$ V. Hannen, ${ }^{8}$ N. Haußmann, ${ }^{14}$ K. Helbing, ${ }^{14}$ S. Hickford, ${ }^{5}$ R. Hiller, ${ }^{5}$ D. Hillesheimer, ${ }^{1}$ D. Hinz,${ }^{6}$ T. Houdy, ${ }^{2,10}$ A. Huber, ${ }^{5}$ A. Jansen, ${ }^{6}$ L. Köllenberger, ${ }^{6}$ C. Karl, ${ }^{2,10}$ J. Kellerer, ${ }^{5}$ L. Kippenbrock, ${ }^{15}$ M. Klein, ${ }^{6,5}$ A. Kopmann, ${ }^{4}$ M. Korzeczek, ${ }^{5}$ A. Kovalík, ${ }^{16}$ B. Krasch, ${ }^{1}$ H. Krause, ${ }^{6}$ T. Lasserre,,${ }^{3,}$ T. L. Le, ${ }^{1}$ O. Lebeda ${ }^{16}$ N. Le Guennic, ${ }^{10}$ B. Lehnert, ${ }^{19}$ A. Lokhov ${ }^{8,7}$ J. M. Lopez Poyato, ${ }^{13}$ K. Müller, ${ }^{6}$ M. Machatschek,${ }^{5}$ E. Malcherek, ${ }^{6}$ M. Mark, ${ }^{6}$ A. Marsteller, ${ }^{1}$ E. L. Martin, ${ }^{11,12}$ C. Melzer, ${ }^{1}$ S. Mertens, ${ }^{2,10}$ S. Niemes, ${ }^{1}$ P. Oelpmann, ${ }^{8}$ A. Osipowicz,${ }^{20}$ D. S. Parno, ${ }^{18}$ A. W. P. Poon, ${ }^{19}$ F. Priester, ${ }^{1}$ M. Röllig, ${ }^{1}$ C. Röttele, ${ }^{1,6,5}$ O. Rest, ${ }^{8}$ R. G. H. Robertson, ${ }^{15}$ C. Rodenbeck, ${ }^{8}$ M. Ryšavý, ${ }^{16}$ R. Sack, ${ }^{8}$ A. Saenz, ${ }^{21}$ A. Schaller, ${ }^{2,10}$ P. Schäfer, ${ }^{1}$ L. Schimpf,${ }^{5}$ M. Schlösser, ${ }^{1}$ K. Schlösser, ${ }^{6}$ L. Schlüter, ${ }^{2,10}$ M. Schrank, ${ }^{6}$ B. Schulz, ${ }^{21}$ M. Šefč́́k, ${ }^{16}$ H. Seitz-Moskaliuk, ${ }^{5}$ V. Sibille, ${ }^{17}$ D. Siegmann, ${ }^{2,10}$ M. Slezák $,{ }^{2}, 10$ F. Spanier, ${ }^{6}$ M. Steidl, ${ }^{6}$ M. Sturm, ${ }^{1}$ M. Sun, ${ }^{15}$ H. H. Telle, ${ }^{13}$ T. Thümmler, ${ }^{6}$ L. A. Thorne, ${ }^{18}$ N. Titov, ${ }^{7}$ I. Tkachev, ${ }^{7}$ N. Trost,${ }^{6}$ D. Vénos, ${ }^{16}$ K. Valerius,${ }^{6}$ A. P. Vizcaya Hernández, ${ }^{18}$ S. Wüstling, ${ }^{4}$ M. Weber, ${ }^{4}$ C. Weinheimer,${ }^{8}$ C. Weiss, ${ }^{22}$ S. Welte, ${ }^{1}$ J. Wendel, ${ }^{1}$ J. F. Wilkerson, ${ }^{11,12}$ J. Wolf, ${ }^{5}$ W. Xu, ${ }^{17}$ Y.-R. Yen, ${ }^{18}$ S. Zadoroghny, ${ }^{7}$ and G. Zeller ${ }^{1}$

(KATRIN Collaboration)

\footnotetext{
${ }^{1}$ Tritium Laboratory Karlsruhe (TLK), Karlsruhe Institute of Technology (KIT),

Hermann-von-Helmholtz-Platz 1, 76344 Eggenstein-Leopoldshafen, Germany

${ }^{2}$ Technische Universität München, James-Franck-Straße 1, 85748 Garching, Germany

${ }^{3}$ IRFU (DPhP and APC), CEA, Université Paris-Saclay, 91191 Gif-sur-Yvette, France

${ }^{4}$ Institute for Data Processing and Electronics (IPE), Karlsruhe Institute of Technology (KIT),

Hermann-von-Helmholtz-Platz 1, 76344 Eggenstein-Leopoldshafen, Germany

${ }^{5}$ Institute of Experimental Particle Physics (ETP), Karlsruhe Institute of Technology (KIT), Wolfgang-Gaede-Straße 1, 76131 Karlsruhe, Germany

${ }^{6}$ Institute for Astroparticle Physics (IAP), Karlsruhe Institute of Technology (KIT),

Hermann-von-Helmholtz-Platz 1, 76344 Eggenstein-Leopoldshafen, Germany

${ }^{7}$ Institute for Nuclear Research of Russian Academy of Sciences, 60th October Anniversary Prospect 7a, 117312 Moscow, Russia

${ }^{8}$ Institut für Kernphysik, Westfälische Wilhelms-Universität Münster, Wilhelm-Klemm-Straße 9, 48149 Münster, Germany

${ }^{9}$ Max-Planck-Institut für Kernphysik, Saupfercheckweg 1, 69117 Heidelberg, Germany

${ }^{10}$ Max-Planck-Institut für Physik, Föhringer Ring 6, 80805 München, Germany

${ }^{11}$ Department of Physics and Astronomy, University of North Carolina, Chapel Hill, North Carolina 27599, USA

${ }^{12}$ Triangle Universities Nuclear Laboratory, Durham, North Carolina 27708, USA

${ }^{13}$ Departamento de Química Física Aplicada, Universidad Autonoma de Madrid, Campus de Cantoblanco, 28049 Madrid, Spain

${ }^{14}$ Department of Physics, Faculty of Mathematics and Natural Sciences, University of Wuppertal, Gaußstraße 20, 42119 Wuppertal, Germany

${ }^{15}$ Center for Experimental Nuclear Physics and Astrophysics, and Deptartment of Physics, University of Washington, Seattle, Washington 98195, USA

${ }^{16}$ Nuclear Physics Institute of the CAS, v. v. i., CZ-250 68 Řez, Czech Republic

${ }^{17}$ Laboratory for Nuclear Science, Massachusetts Institute of Technology, 77 Massachusetts Ave, Cambridge, Massachusetts 02139, USA

${ }^{18}$ Department of Physics, Carnegie Mellon University, Pittsburgh, Pennsylvania 15213, USA

${ }^{19}$ Institute for Nuclear and Particle Astrophysics and Nuclear Science Division, Lawrence Berkeley National Laboratory, Berkeley, California 94720, USA

${ }^{20}$ University of Applied Sciences (HFD) Fulda, Leipziger Straße 123, 36037 Fulda, Germany

${ }^{21}$ Institut für Physik, Humboldt-Universität zu Berlin, Newtonstraße 15, 12489 Berlin, Germany
} 


\title{
${ }^{22}$ Project, Process, and Quality Management (PPQ), Karlsruhe Institute of Technology (KIT), Hermann-von-Helmholtz-Platz, 1, 76344 Eggenstein-Leopoldshafen, Germany
}

(Received 13 November 2020; revised 6 January 2021; accepted 22 January 2021; published 5 March 2021)

\begin{abstract}
We report on the light sterile neutrino search from the first four-week science run of the KATRIN experiment in 2019. Beta-decay electrons from a high-purity gaseous molecular tritium source are analyzed by a high-resolution MAC-E filter down to $40 \mathrm{eV}$ below the endpoint at $18.57 \mathrm{keV}$. We consider the framework with three active neutrinos and one sterile neutrino. The analysis is sensitive to the mass, $m_{4}$, of the fourth mass state for $m_{4}^{2} \lesssim 1000 \mathrm{eV}^{2}$ and to active-to-sterile neutrino mixing down to $\left|U_{e 4}\right|^{2} \gtrsim 2 \times 10^{-2}$. No significant spectral distortion is observed and exclusion bounds on the sterile mass and mixing are reported. These new limits supersede the Mainz results for $m_{4}^{2} \lesssim 1000 \mathrm{eV}^{2}$ and improve the Troitsk bound for $m_{4}^{2}<30 \mathrm{eV}^{2}$. The reactor and gallium anomalies are constrained for $100<\Delta m_{41}^{2}<1000 \mathrm{eV}^{2}$.
\end{abstract}

DOI: 10.1103/PhysRevLett.126.091803

Introduction.-Neutrino measurements in the three flavor framework have determined all mixing angles and mass splittings [1]. Neutrino oscillation results from LSND [2] and MiniBooNE [3] suggested experimental evidence for sterile neutrinos, i.e., a nonstandard neutrino that does not interact weakly. In 2011, a reexamination of $\bar{\nu}_{\mathrm{e}}$ emitted from nuclear reactors revealed a significant discrepancy between measured and expected fluxes at $\lesssim 100 \mathrm{~m}$ - the reactor antineutrino anomaly (RAA) [4]. Moreover, both the GALLEX and SAGE experiments reported a deficit of $\nu_{\mathrm{e}}$ from ${ }^{37} \mathrm{Ar}$ and ${ }^{51} \mathrm{Cr}$ electron-capture decays [5-10]- the gallium anomaly (GA). These anomalies are debated, mainly due to the difficulty of assessing systematic uncertainties [11-14]. Nonetheless, this neutrino disappearance could be explained by assuming the existence of a sterile neutrino, with a mass of $\gtrsim 1 \mathrm{eV}$ [15].

The Karlsruhe Tritium Neutrino experiment (KATRIN) [16-19], displayed in Fig. 1, provides high-precision electron spectrum measurement of tritium $\beta$ decay, ${ }^{3} \mathrm{H} \rightarrow{ }^{3} \mathrm{He}^{+}+$ $e^{-}+\bar{\nu}_{\mathrm{e}}$ (endpoint $E_{0}=18.57 \mathrm{keV}$, half-life $t_{1 / 2}=12.32 \mathrm{yr}$ ). KATRIN is designed to improve the sensitivity on the effective neutrino mass, $m_{\nu}$, to $0.2 \mathrm{eV}$ (90\% C.L.). Based on its first four-week science run in spring 2019, KATRIN reported $m_{\nu}^{2}=\left(-1.0_{-1.1}^{+0.9}\right) \mathrm{eV}^{2}$, leading to $m_{\nu}<1.1 \mathrm{eV}$ (90\% C.L.) [20]. Using the same data set, one can limit the mass and flavor composition of a fourth neutrino mass state that would manifest itself as a distortion of the $\beta$-electron spectrum. The signature would be a kinklike feature, as shown in a simulation presented in Fig. 2. Previous studies examined the sensitivity of KATRIN to sterile neutrinos [21-23]. The authors of [24] reported limits based

Published by the American Physical Society under the terms of the Creative Commons Attribution 4.0 International license. Further distribution of this work must maintain attribution to the author(s) and the published article's title, journal citation, and DOI. Funded by SCOAP. on publicly accessible KATRIN data that do not contain all the necessary inputs to perform a comprehensive analysis, however. Here, we report the first search for light sterile neutrinos by KATRIN.

Experimental setup.-KATRIN combines a windowless gaseous molecular tritium source (WGTS) [25], with a spectrometer section based on the principle of magnetic adiabatic collimation with electrostatic filtering (MAC-Efilter) [26-29]. Figure 1 gives an overview of the $70 \mathrm{~m}$ long experimental setup located at the Karlsruhe Institute of Technology in Germany. High-purity tritium gas is continuously injected at $30 \mathrm{~K}$ into the WGTS in a closed loop [30]. Then, the gas diffuses to the ends of the source where it is pumped out by a series of turbomolecular pumps. In combination with the $3 \mathrm{~K}$ cryotrap, the flow rate of tritium into the spectrometer-detector sections downstream [Figs. 1(d) $-1(\mathrm{f})$ ] is reduced by $>14$ orders of magnitude to suppress source-related background [16]. Electrons are adiabatically guided toward the spectrometers by the source magnetic field $\left(B_{\mathrm{WGTS}}=2.52 \mathrm{~T}\right)$ and other superconducting magnets [31] in the pumping section. High-precision spectroscopy is achieved by the MAC-E-filter, where electrons of charge $q$ are guided by the magnetic field and filtered by an electrostatic retarding potential energy, $q U$, set by a specific high voltage (HV) setting. Only electrons with energies larger than $q U$ are transmitted. By varying and monitoring $q U$ the $\beta$-electron spectrum is scanned in an integral mode, with an energy resolution $\Delta E=2.8 \mathrm{eV}$ at $E_{0}$. Transmitted electrons are counted in a 148-pixel silicon detector. [32].

Measurement.-The performance of the KATRIN systems [17] was established by a sequence of long-term measurements [18,30,31,33,34]. Here, we use the data from KATRIN's first high-purity tritium campaign, which ran from April 10 to May 13, 2019, at an average source activity of $2.45 \times 10^{10} \mathrm{~Bq}$. The averaged column density $\rho d_{\text {exp }}=1.11 \times 10^{17}$ molecules $\mathrm{cm}^{-2}$ of this data sample is a factor of 5 below its nominal value. The isotopic tritium 


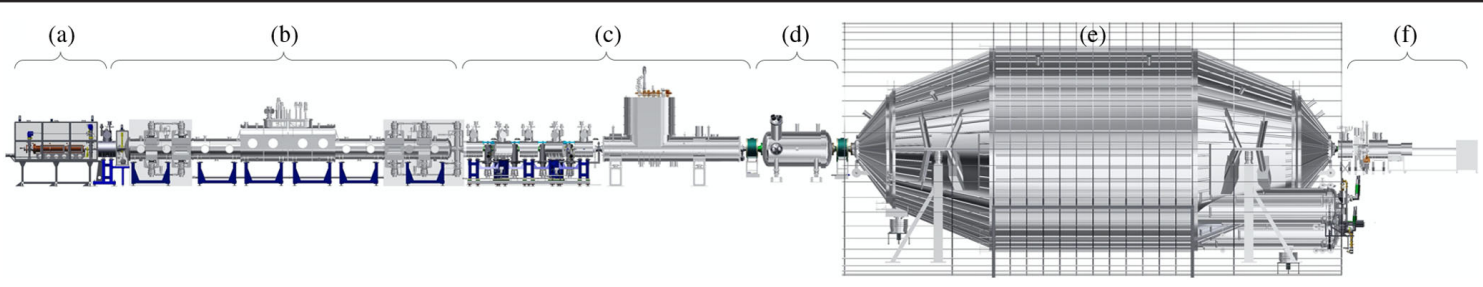

FIG. 1. Components of the KATRIN experiment: (a) the rear section, (b) the windowless gaseous tritium source, (c) the pumping section, (d) the prespectrometer, (e) the main spectrometer, (f) the electron detector.

purity $\varepsilon_{\mathrm{T}}(0.976)$ is derived from the average concentration of the tritiated species $\mathrm{T}_{2}$ (0.953), HT (0.035), and DT (0.011), continuously monitored using Raman spectroscopy [35].

The integral $\beta$-electron spectrum is scanned repeatedly in the range of [ $E_{0}-90$ to $\left.E_{0}+50 \mathrm{eV}\right]$ by applying nonequidistant $\mathrm{HV}$ settings to the spectrometer electrode system. Each scan lasted $2 \mathrm{~h}$. At each HV set point, the transmitted electrons are counted over time intervals varying from 17 to $576 \mathrm{~s}$. We analyze the scan range from $40 \mathrm{eV}$ below $E_{0}$ (22 $\mathrm{HV}$ set points) to $50 \mathrm{eV}$ above (5 HV set points). Figure 2(c) shows the measurement time distribution.

Data analysis.-First, we apply quality cuts to slowcontrol parameters associated with each scan (27 HV set points). This results in the selection of 274 stable scans with an overall scanning time of $521.7 \mathrm{~h}$. Then, we select the 117 best pixels (79\% of the sensitive area of the detector) and combine them into a single effective pixel [36]. The temporal stability of the scanning process, verified by fits of the 274 single-scan $\beta$-decay endpoints, allows us to stack the data from these scans into a single $90-\mathrm{eV}$-wide spectrum displayed in Fig. 2(a). The resulting stacked integral spectrum, $R(\langle q U\rangle)$, includes $2.03 \times 10^{6}$ events, with $1.48 \times 10^{6} \beta$ electrons expected below $E_{0}$ and a flat background ensemble of $0.55 \times 10^{6}$ events in the whole scan interval. This background originates from two main sources in the spectrometer: first, the thermal ionization of Rydberg atoms sputtered off the inner spectrometer surfaces by ${ }^{206} \mathrm{~Pb}$-recoil ions following $\alpha$ decays of ${ }^{210} \mathrm{Po}$; second, the secondary electrons induced by $\alpha$ decays of single ${ }^{219} \mathrm{Rn}$ atoms emanating from the vacuum pumps. The resulting sub-eV electrons are accelerated to $q U$ by the MAC-E-filter. The radon-induced background is nonPoissonian (see [20]). Nonetheless, in comparison to reactor neutrino experiments with baselines of less than 15 meters [37-39], our search has a high signal-tobackground ratio, rapidly increasing from 1 at $\langle q U\rangle=$ $E_{0}-12 \mathrm{eV}$ to $>70$ at $\langle q U\rangle=E_{0}-40 \mathrm{eV}$.

Modeling.-The modeled experimental spectrum $R_{\text {calc }}(\langle q U\rangle)$ is the convolution of the differential $\beta$ spectrum $R_{\beta}(E)$ with the response function $f(E-\langle q U\rangle)$, and an energy-independent background rate $R_{\mathrm{bg}}$

$$
R_{\text {calc }}(\langle q U\rangle)=A_{\mathrm{s}} N_{\mathrm{T}} \int R_{\beta}(E) f(E-\langle q U\rangle) d E+R_{\mathrm{bg}},
$$

where $E$ is the electron kinetic energy, and $A_{s}$ is the tritium signal amplitude. $N_{\mathrm{T}}$ denotes the number of tritium atoms in the source multiplied with the accepted solid angle of the setup $\Delta \Omega / 4 \pi=\left(1-\cos \theta_{\max }\right) / 2$, with $\theta_{\max }=50.4^{\circ}$, and the detector efficiency (0.95).

For superallowed $\beta$ decay of molecular tritium

$$
\begin{aligned}
R_{\beta}(E)= & \frac{G_{F}^{2} \cos ^{2} \Theta_{C}}{2 \pi^{3}}\left|M_{\text {nucl }}^{2}\right| F\left(E, Z^{\prime}\right) \\
& \times\left(E+m_{e}\right) \sqrt{\left(E+m_{e}\right)^{2}-m_{e}{ }^{2}} \\
& \times \sum_{\mathrm{j}} \zeta_{j} \varepsilon_{j} \sqrt{\varepsilon_{j}^{2}-m_{\nu}^{2}} \Theta\left(\varepsilon_{j}-m_{\nu}\right),
\end{aligned}
$$
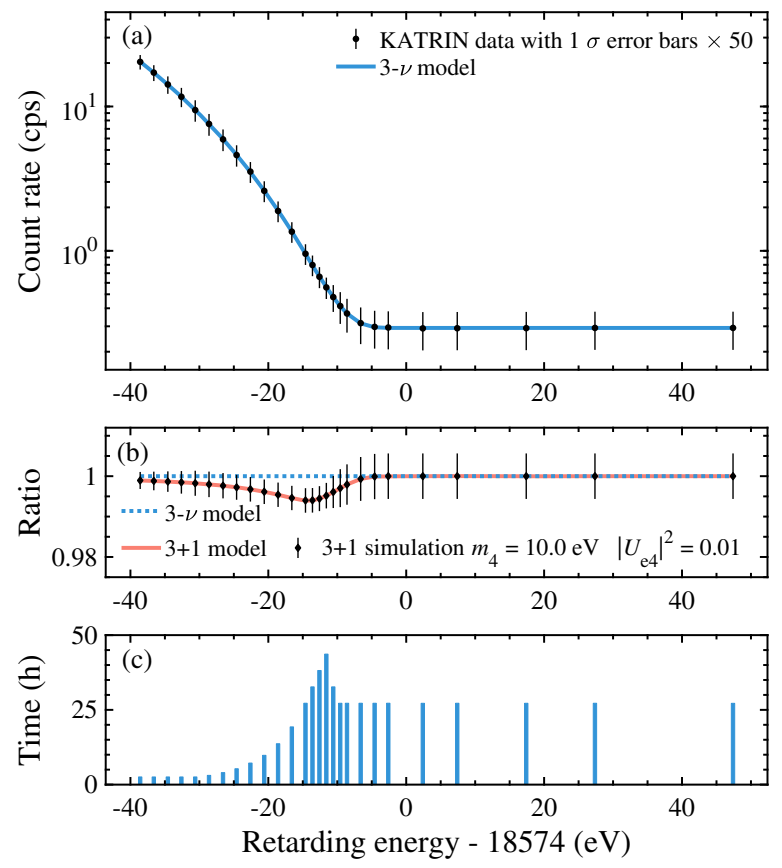

FIG. 2. (a) Electron spectrum of experimental data $R(\langle q U\rangle)$ over the interval $\left[E_{0}-40\right.$ to $\left.E_{0}+50 \mathrm{eV}\right]$ from all 274 tritium scans and the three-neutrino mixing best-fit model $R_{\text {calc }}(\langle q U\rangle)$ (line). The integral $\beta$-decay spectrum extends to $E_{0}$ on top of a flat background $R_{\mathrm{bg}}$. The rate is given in counts per second (cps). $1 \sigma$ errors are enlarged by a factor 50. (b) Simulation of an arbitrary sterile neutrino imprint on electron spectrum. The ratio of the simulated data without fluctuation, including a fourth neutrino of mass $m_{4}=10 \mathrm{eV}$ and mixing $\left|U_{e 4}\right|^{2}=0.01$, to the three-neutrino mixing model is shown (red solid line). (c) Integral measurement time distribution of all $27 \mathrm{HV}$ set points. 
with the square of the energy-independent nuclear matrix element $\left|M_{\text {nucl }}^{2}\right|$, the neutrino energy $\varepsilon_{j}=E_{0}-E-V_{j}$, the Fermi constant $G_{F}$, the Cabibbo angle $\Theta_{C}$, the electron mass $m_{e}$, and the Fermi function $F\left(E, Z^{\prime}=2\right)$. The calculation of $R_{\beta}(E)$ involves the sum over a final-state distribution given by the probabilities $\zeta_{j}$ that the daughter ion ${ }^{3} \mathrm{HeT}^{+}$is left in a molecular (i.e., a rotational, vibrational, and electronic) state with excitation energy $V_{j}$ [20]. In our calculations, we have included radiative corrections [19] and Doppler broadening at $30 \mathrm{~K}$.

The function $f(E-\langle q U\rangle)$ describes the transmission probability of an electron as a function of its surplus energy $E-\langle q U\rangle$. It depends on the angular spread of electrons and the amount of neutral gas they pass through in the source, where they can undergo inelastic scattering [20].

In the three-neutrino framework $m_{\nu}^{2}=\sum_{k=1}^{3}\left|U_{e k}\right|^{2} m_{k}^{2}$, where $U$ is the $3 \times 3$ Pontecorvo-Maki-Nakagawa-Sakata unitary mixing matrix and $m_{k}$ the eigenvalue of mass state $k(=1,2,3)$. In this framework, later referred to as the null hypothesis, the experimental spectrum $R(\langle q U\rangle)$ is well described by the model of the response function $f$ and by the background $R_{\mathrm{bg}}=(293 \pm 1)$ mcps mainly constrained by the $5 \mathrm{HV}$ set points above $E_{0}$.

Sterile neutrino search.-We extend the experimental modeling and statistical analysis to constrain both the sterile neutrino mass squared $m_{4}^{2}$ and its mixing amplitude $\left|U_{e 4}\right|^{2}$, following the same strategy as for our $m_{\nu}$ analysis [20].

In the $3+1$ active-sterile neutrino model extension, $m_{\nu}^{2}$ can be redefined as $m_{\nu}^{2}=\sum\left|U_{e k}\right|^{2} m_{k}^{2}\left(1-\left|U_{e 4}\right|^{2}\right)^{-1}$. The electron spectrum, $R_{\beta}$, is replaced by $R_{\beta}\left(E, m_{\nu}, m_{4}\right)=$ $\left(1-\left|U_{e 4}\right|^{2}\right) R_{\beta}\left(E, m_{\nu}^{2}\right)+\left|U_{e 4}\right|^{2} R_{\beta}\left(E, m_{4}^{2}\right)$, where $U$ is the extended $4 \times 4$ unitary matrix, $R_{\beta}\left(E, m_{\nu}^{2}\right)$ is the differential electron spectrum [Eq. (2)] associated with decays that include active neutrinos in the final state, and $R_{\beta}\left(E, m_{4}^{2}\right)$ describes the additional spectrum associated to decays involving a fourth neutrino (mostly sterile) of mass $m_{4}$. The observable integral spectrum $R_{\text {calc }}$ is, henceforth, modeled with six free parameters: the four original parameters $\left(A_{s}, E_{o}, R_{b g}, m_{\nu}^{2}\right)[20], m_{4}^{2}$, and $\left|U_{e 4}\right|^{2}$. This extended model $R_{\text {calc }}(\langle q U\rangle)$ is then fitted to the experimental data $R(\langle q U\rangle)$. In order to mitigate bias, the full analysis is, first, conducted on a Monte Carlo (MC) data set before turning to the actual data without any modification. For each experimental scan $k$, we generate a "MC twin," $R_{\text {calc }}(\langle q U\rangle)_{k}$, from its averaged slow-control parameters and the measured background rate and endpoint. The MC twin analysis allows us to verify the accuracy of our parameter inference by reproducing the input MC values. This approach is also used to calculate the expected sensitivity and to assess the impact of each systematic uncertainty, described in detail in [20].

The fit of $R(\langle q U\rangle)$ with $R_{\text {calc }}(\langle q U\rangle)$ is performed by minimizing the standard $\chi^{2}$ estimator. In a "shape-only" fit, both $E_{0}$ and $A_{s}$ are left unconstrained. To propagate systematic uncertainties, a covariance matrix is computed after performing $\mathcal{O}\left(10^{4}\right)$ simulations of $R_{\text {calc }}(\langle q U\rangle)$ while varying parameters according to their likelihood in each calculation [20,40-42]. The sum of all matrices encodes the total uncertainties of $R_{\text {calc }}(\langle q U\rangle)$, including HV set-pointdependent correlations. The $\chi^{2}$ estimator is then minimized to determine the best-fit parameters, and the shape of the $\chi^{2}$ function is used to infer the uncertainties.

To obtain the sterile neutrino constraints, fits are performed on a $50 \times 50\left[\log \left(\left|U_{e 4}\right|^{2}\right), \log \left(m_{4}^{2}\right)\right]$ grid (starting with the null hypothesis), by keeping $\left|U_{e 4}\right|^{2}$ and $m_{4}^{2}$ constant while minimizing $\chi^{2}$ with respect to all other free parameters. A finer grid does not significantly change our results. The $95 \%$ C.L. is given by the contour given by $\Delta \chi^{2}=\chi^{2}-\chi_{\min }^{2}=5.99$, assuming Wilks' theorem [43] for 2 degrees of freedom. $\chi_{\min }^{2}$ is the global minimum of all $\chi^{2}$ values obtained in the grid scan. We have verified that the global minimum lies within the physical region defined as $\left|U_{e 4}\right|^{2} \in[0,0.5]$ and $m_{4}^{2} \geq 0$, by enlarging the grid scan to the nonphysical regions. The coverage of this approach is validated by simulations, where thousands of experiments were generated for the null hypothesis and a few sterile neutrino signal hypotheses and analyzed in turn.

Results. -The fit range $\left[E_{0}-40\right.$ to $\left.E_{0}+50 \mathrm{eV}\right]$ is chosen such that statistical uncertainties on $\left|U_{e 4}\right|^{2}$ dominate over systematic uncertainties, described in $[20,36]$. Over the whole range of $m_{4}^{2}$ considered, we have $\sigma_{\text {sys }}^{2}<0.1 \sigma_{\text {stat }}^{2}$.

In our main analysis, labeled case I, we consider the hierarchical scenario $m_{1,2,3} \ll m_{4}$, which justifies setting $m_{\nu}$ to its minimum allowable value. Here, we set $m_{\nu}$ to zero, which is consistent within our sensitivity with the lower limit derived from neutrino oscillations $(0.009 \mathrm{eV}$, see [1]).

For each $\left(\left|U_{e 4}\right|^{2}, m_{4}^{2}\right)$ pair, a fit compares the experimental $R(\langle q U\rangle)$ to the model $R_{\text {calc }}(\langle q U\rangle)$ by only considering $A_{\text {sig }}, R_{\mathrm{bg}}, E_{0}$, as free parameters. The global best fit minimum is found for $m_{4}^{2}=73.0 \mathrm{eV}^{2}$, and $\left|U_{e 4}\right|^{2}=0.034$. The $\chi^{2}$ difference between this best fit and the null hypothesis is $\Delta \chi_{\mathrm{bf}}^{2}=1.6$. Assuming the null hypothesis, the probability to obtain $\Delta \chi_{\text {bf }}^{2} \geq 1.6$ is $50 \%$, based on the simulation and analysis of 2000 pseudoexperiments. Therefore, our result is consistent with the null-hypothesis hypothesis that there is no evidence for a sterile neutrino signal. The resulting 95\% C.L. exclusion and sensitivity curves are shown in Fig. 3. Our results agree well with the sensitivity estimates. Since the data cover the last $40 \mathrm{eV}$ of the $\beta$ spectrum, this analysis is only sensitive to $m_{4}^{2}<$ $1600 \mathrm{eV}^{2}$ with a maximum sensitivity at $m_{4}^{2} \simeq 400 \mathrm{eV}^{2}$. For smaller $m_{4}^{2}$, the sensitivity decreases due to the reduction of statistics and vanishes for $m_{4}^{2} \simeq 2 \mathrm{eV}^{2}$. For larger $m_{4}^{2}$, the sensitivity rapidly drops due to the narrowing interval in which a sterile neutrino could influence the measured $\beta$ spectrum. Case I allows a direct comparison with previous experiments. This Letter supersedes the 
Mainz exclusion limit [44] for $m_{4}^{2} \lesssim 1000 \mathrm{eV}^{2}$ and improves the Troitsk bounds [45] for $m_{4}^{2} \lesssim 30 \mathrm{eV}^{2}$, as displayed in Fig. 3.

In the second analysis, called case II, $m_{\nu}^{2}$ is treated as an unconstrained parameter. Figure 3 shows the resulting $95 \%$ C.L. exclusion curves in the $\left(\left|U_{e 4}\right|^{2}, m_{4}^{2}\right)$ plane, only deviating from the upper limit of case I for $m_{4}^{2}<60 \mathrm{eV}^{2}$. For low mixing, $\left|U_{e 4}\right|^{2} \lesssim 0.3$, and small $m_{4}^{2}<10 \mathrm{eV}^{2}$, the fitted $m_{\nu}^{2}$ values are within $1 \sigma$ of the value obtained in our neutrino mass analysis [20]. Figure 3 also shows the exclusion curve of a similar analysis, denoted case III, with $m_{\nu}^{2}$ treated as a nuisance parameter constrained by a Gaussian pull term with the expectation $m_{\nu}^{2}=0 \mathrm{eV}^{2}$ and $\sigma\left(m_{\nu}^{2}\right)=1 \mathrm{eV}^{2}$.

Comparison with neutrino oscillation experiments.-It is interesting to compare our case I results with short baseline $\nu$-oscillation experiments measuring the electron (anti) neutrino survival probability $P\left[\Delta m_{41}^{2}, \sin ^{2}\left(2 \theta_{e e}\right)\right]$ [15]. To relate those results to KATRIN, the mass splitting can be written as $\Delta m_{41}^{2} \simeq m_{4}^{2}-m_{\nu}^{2}$. This approximation is valid to within $2 \times 10^{-4} \mathrm{eV}^{2}$ [24]; For case I, we simply have $\Delta m_{41}^{2} \simeq m_{4}^{2}$. Furthermore, KATRIN is directly sensitive to $\left|U_{e 4}\right|^{2}$, whereas oscillation experiments measure $\sin ^{2}\left(2 \theta_{e e}\right)=4\left|U_{e 4}\right|^{2}\left(1-\left|U_{e 4}\right|^{2}\right)$. Our results exclude $\Delta m_{41}^{2}$ between 100 and $1000 \mathrm{eV}^{2}$ - the high $\Delta m_{41}^{2}$ solution

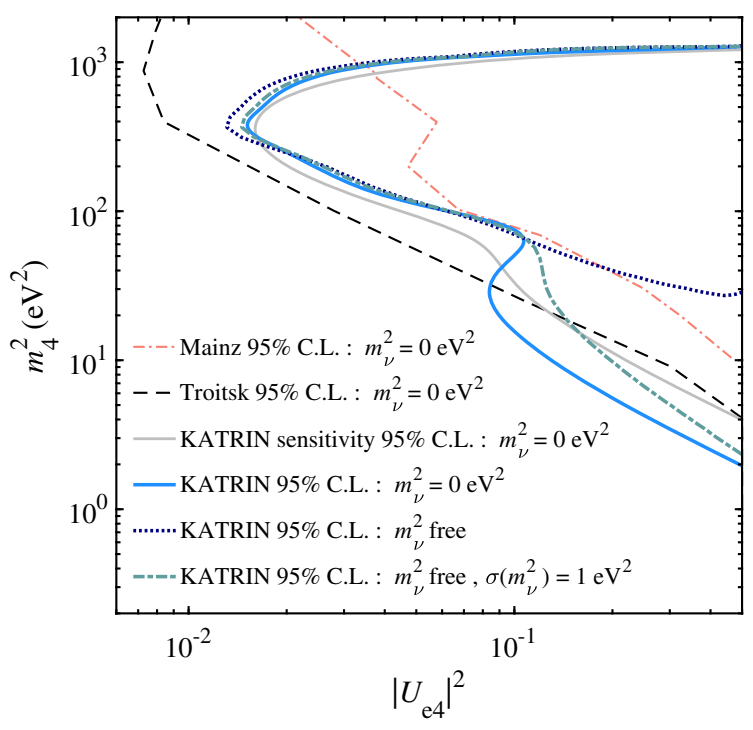

FIG. 3. $95 \%$ C.L. exclusion curves in the $\left(\left|U_{e 4}\right|^{2}, m_{4}^{2}\right)$ plane obtained from this analysis. Both statistical and systematic uncertainties are included. The two solid lines show the expected sensitivity (light gray) and the associated exclusion (blue) for fixed $m_{\nu}^{2}=0 \mathrm{eV}^{2}$ (case I). The dotted line in dark blue illustrates the exclusion curve obtained with a free $m_{\nu}^{2}$ (case II). Last, the dot-dashed line in turquoise displays the intermediate exclusion curve with a free $m_{\nu}^{2}$ constrained with an uncertainty $\sigma\left(m_{\nu}^{2}\right)=$ $1 \mathrm{eV}^{2}$ (case III). These results supersede the Mainz exclusion limit [44] for $m_{4}^{2} \lesssim 1000 \mathrm{eV}^{2}$ and improve the Troitsk bounds [45] for $m_{4}^{2}<30 \mathrm{eV}^{2}$. for GA and RAA - as depicted in Fig. 4. Our results also strengthen the exclusion of $\Delta m_{41}^{2} \gtrsim 10 \mathrm{eV}^{2}$, achieved previously by the DANSS, PROSPECT, and STEREO reactor spectral ratio measurements $[37,39,46]$. The hint of large active-sterile mixing in Neutrino 4 [47] is at the edge of our current $95 \%$ C.L. exclusion. In Fig. 4, we also compare our result to medium baseline reactor $\bar{\nu}_{e}$ disappearance experiments $[48,49]$. Results of experiments sensitive to other mixing angles, such as $\theta_{\mu e}$ in the case of the Daya Bay and MINOS + combination [48], are not displayed here.

An estimation of KATRIN's five-year sensitivity is presented in Fig. 4, assuming 1000 live days of data at the nominal column density, the current reduced background (130 mcps), and design uncertainties [17]. KATRIN results will be complementary to short baseline reactor neutrino experiments, improving the global sensitivity for $\Delta m_{41}^{2} \gtrsim 5 \mathrm{eV}^{2}$.

If sterile neutrinos with $\left|U_{e 4}\right| \neq 0$ are Majorana particles, they will contribute to the effective mass $m_{\beta \beta}=\left|\sum U_{e i}^{2} m_{i}\right|$

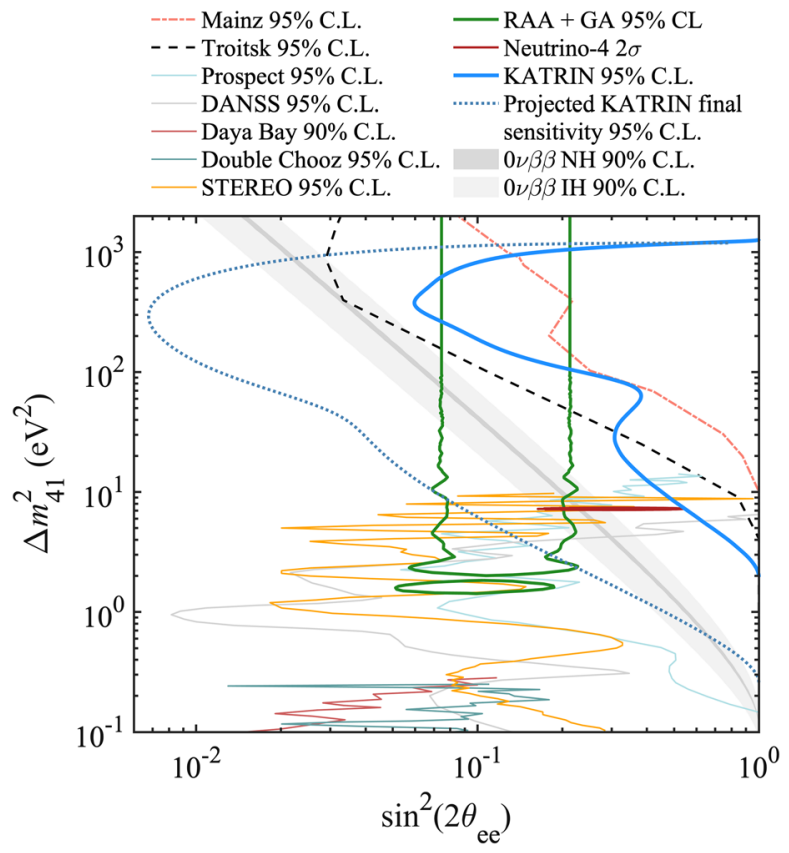

FIG. 4. $95 \%$ C.L. exclusion curves in the $\left(\sin ^{2}\left(2 \theta_{e e}\right), \Delta m_{41}^{2}\right)$ plane obtained from the analysis of KATRIN data with fixed $m_{\nu}=0$. The green contour delimits the $3+1$ neutrino oscillations allowed at $95 \%$ C.L. by the reactor and gallium anomalies [4]. KATRIN data improve the exclusion of the high $\Delta m_{41}^{2}$ values with respect to DANSS, PROSPECT, STEREO, Daya Bay, and Double Chooz reactor measurements [37,39,46,48,49]. Mainz [44] and Troitsk [45] exclusion curves [50] are also displayed for comparison. An estimation of KATRIN's final sensitivity is represented by the dotted line. The light (dark) gray bands delimit the exclusions from $0 \nu \beta \beta$ experiments, for the case of inverted and normal hierarchies (the extension of the bands reflects the uncertainties of the parameters of the Pontecorvo-Maki-Nakagawa-Sakata matrix [1]). 
relevant for $0 \nu \beta \beta$ [4,51,52]. Considering $m_{4} \gg m_{1,2,3}$, we have the active neutrino contribution to $m_{\beta \beta}$ within the ranges 0.01 to $0.05 \mathrm{eV}$ ( 0 to $0.005 \mathrm{eV}$ ) for the inverted (normal) ordering. Our current and future constraints on $U_{e 4}$ and $m_{4}$ can then be confronted with the latest constraints of $0 \nu \beta \beta$ experiments [53,54], as shown in Fig. 4.

Conclusion and outlook.-We have presented a search for signatures of a sterile neutrino admixture $(3+1$ framework) using data from the first KATRIN science run. This search comprises $1.48 \times 10^{6} \beta$ electrons and $0.41 \times 10^{6}$ background events below $E_{0}$, with a signalto-background ratio of up to 70 . The analysis is sensitive to $m_{4}$ ranging from about 2 to $40 \mathrm{eV}$. No significant sterile neutrino signal is observed and exclusion limits on the parameters $\left|U_{e 4}\right|^{2}$ and $m_{4}$ are obtained. Our best sensitivity is for $m_{4} \sim 20 \mathrm{eV}$, excluding $\left|U_{e 4}\right|^{2} \gtrsim 2 \times 10^{-2}$. Our result improves bounds set by previous direct kinematic experiments. This search is complementary to reactor oscillation experiments and improves their constraints for $\Delta m_{41}^{2} \gtrsim 10 \mathrm{eV}^{2}$, excluding a fraction of the allowed GA and RAA parameter space. KATRIN will significantly improve its statistics in the next five years and further reduce its systematics and background enabling the search in a larger fraction of the GA and RAA region.

We acknowledge the support of Helmholtz Association, Ministry for Education and Research BMBF (Grants No. 5A17PDA, No. 05A17PM3, No. 05A17PX3, No. 05A17VK2, and No. 05A17WO3), Helmholtz Alliance for Astroparticle Physics (HAP), Helmholtz Young Investigator Group (Grant No. VH-NG-1055), and Deutsche Forschungsgemeinschaft DFG (Research Training Groups GRK 1694 and GRK 2149, and Graduate School GSC 1085-KSETA) in Germany; Ministry of Education, Youth and Sport (Grants No. CANAM-LM2011019, No. LTT19005) in the Czech Republic; Ministry of Science and Higher Education of the Russian Federation under Contract No. 075-15-2020-778; and the United States Department of Energy through Grants No. DE-FG02-97ER41020, No. DE-FG02-94ER40818, No. DE-SC0004036, No. DE-FG02-97ER41033, No. DE-FG02-97ER41041, No. DE-AC02-05CH11231, No. DE-SC0011091, and No. DE-SC0019304, and the National Energy Research Scientific Computing Center.

* Corresponding author. thierry.lasserre@cea.fr

[1] P. A. Zyla et al. (Particle Data Group), Prog. Theor. Exp. Phys. 2020, 083C01 (2020).

[2] A. Aguilar et al., Phys. Rev. D 64, 112007 (2001).

[3] A. Aguilar-Arevalo et al., Phys. Rev. Lett. 121, 221801 (2018).

[4] G. Mention, M. Fechner, T. Lasserre, T. A. Mueller, D. Lhuillier, M. Cribier, and A. Letourneau, Phys. Rev. D 83, 073006 (2011).
[5] P. Anselmann et al. (GALLEX Collaboration), Phys. Lett. B 342, 440 (1995).

[6] W. Hampel et al. (GALLEX Collaboration), Phys. Lett. B 420, 114 (1998).

[7] F. Kaether, W. Hampel, G. Heusser, J. Kiko, and T. Kirsten, Phys. Lett. B 685, 47 (2010).

[8] D. Abdurashitov et al. (SAGE Collaboration), Phys. Rev. Lett. 77, 4708 (1996).

[9] J. Abdurashitov et al. (SAGE Collaboration), Phys. Rev. C 73, 045805 (2006).

[10] J. Abdurashitov et al. (SAGE Collaboration), Phys. Rev. C 80, 015807 (2009).

[11] T. Mueller et al., Phys. Rev. C 83, 054615 (2011).

[12] P. Huber, Phys. Rev. C 84, 024617 (2011); 85, 029901(E) (2012).

[13] J. M. Berryman and P. Huber, Phys. Rev. D 101, 015008 (2020).

[14] J. Kostensalo, J. Suhonen, C. Giunti, and P. Srivastava, Phys. Lett. B 795, 542 (2019).

[15] C. Giunti and T. Lasserre, Annu. Rev. Nucl. Part. Sci. 69, 163 (2019).

[16] A. Osipowicz et al. (KATRIN Collaboration), arXiv:hep-ex/ 0109033.

[17] C. Giunti, Y. Li, and Y. Zhang, J. High Energy Phys. 05 (2020) 061.

[18] M. Arenz et al. (KATRIN Collaboration), J. Instrum. 13, P04020 (2018).

[19] M. Kleesiek et al., Eur. Phys. J. C 79, 204 (2019).

[20] M. Aker et al. (KATRIN Collaboration), Phys. Rev. Lett. 123, 221802 (2019).

[21] A. S. Riis and S. Hannestad, J. Cosmol. Astropart. Phys. 02 (2011) 011.

[22] J. Formaggio and J. Barrett, Phys. Lett. B 706, 68 (2011).

[23] A. Esmaili and O. L. G. Peres, Phys. Rev. D 85, 117301 (2012).

[24] C. Giunti, Y. Li, and Y. Zhang, J. High Energy Phys. 05 (2020) 61.

[25] R. G. H. Robertson, T. J. Bowles, G. J. Stephenson, D. L. Wark, J. F. Wilkerson, and D. A. Knapp, Phys. Rev. Lett. 67, 957 (1991).

[26] V. M. Lobashev and P. E. Spivak, Nucl. Instrum. Methods Phys. Res., Sect. A 240, 305 (1985).

[27] A. Picard et al., Nucl. Instrum. Methods Phys. Res., Sect. B 63, 345 (1992).

[28] C. Kraus et al., Eur. Phys. J. C 40, 447 (2005).

[29] V. N. Aseev et al., Phys. Rev. D 84, 112003 (2011).

[30] F. Priester, M. Sturm, and B. Bornschein, Vacuum 116, 42 (2015).

[31] M. Arenz et al. (KATRIN Collaboration), J. Instrum. 13, T08005 (2018).

[32] J. F. Amsbaugh et al., Nucl. Instrum. Methods Phys. Res., Sect. A 778, 40 (2015).

[33] M. Arenz et al., Eur. Phys. J. C 78, 368 (2018).

[34] K. Altenmüller et al., J. Phys. G 47, 065002 (2020).

[35] M. Aker et al. (KATRIN Collaboration), Sensors 20, 4827 (2020).

[36] M. Aker et al. (KATRIN Collaboration), arXiv:2101.05253.

[37] M. Danilov (DANSS Collaboration), in Proceedings of the European Physical Society Conference on High 
Energy Physics, EPS-HEP2019, 2019, Ghent, Belgium (2019), https://doi.org/10.22323/1.364.0401.

[38] J. Ashenfelter et al. (PROSPECT Collaboration), Phys. Rev. Lett. 121, 251802 (2018).

[39] H. Almazán et al. (STEREO Collaboration), Phys. Rev. D 102, 052002 (2020).

[40] R. J. Barlow, Statistics: A Guide to the Use of Statistical Methods in the Physical Sciences, Manchester Physics Series (Wiley, Chichester, 1989).

[41] G. D’Agostini, Nucl. Instrum. Methods Phys. Res., Sect. A 346, 306 (1994).

[42] M. Aker et al. (KATRIN Collaboration), Eur. Phys. J. C 80, 264 (2020).

[43] S. Wilks, Ann. Math. Stat. 9, 60 (1938).

[44] C. Kraus, A. Singer, K. Valerius, and C. Weinheimer, Eur. Phys. J. C 73, 2323 (2013).

[45] A. I. Belesev, A. I. Berlev, E. V. Geraskin, A. A. Golubev, N. A. Likhovid, A. A. Nozik, V. S. Pantuev, V. I. Parfenov, and A. K. Skasyrskaya, JETP Lett. 97, 67 (2013).
[46] M. Andriamirado et al. (PROSPECT Collaboration), Phys. Rev. D 103, 032001 (2021).

[47] A. Serebrov et al. (Neutrino-4 Collaboration), Pis'ma Zh. Eksp. Teor. Fiz. 109, 209 (2019).

[48] P. Adamson et al. (Daya Bay and MINOS + Collaborations), Phys. Rev. Lett. 125, 071801 (2020).

[49] T. Abrahão et al. (Double Chooz Collaboration), arXiv:2009.05515.

[50] C. Giunti, M. Laveder, Y. F. Li, and H. W. Long, Phys. Rev. D 87, 013004 (2013).

[51] J. Barry, W. Rodejohann, and H. Zhang, J. High Energy Phys. 07 (2011) 091.

[52] C. Giunti and E. Zavanin, J. High Energy Phys. 07 (2015) 171.

[53] A. Gando et al. (KamLAND-Zen Collaboration), Phys. Rev. Lett. 117, 082503 (2016); 117, 109903(A) (2016).

[54] M. Agostini et al. (GERDA Collaboration), Phys. Rev. Lett. 125, 252502 (2020). 